\title{
Multi-objective production planning model for equipment manufacturing enterprises with multiple uncertainties in demand
}

\author{
Liu, Y.F. ${ }^{a, b,{ }^{*}}$, Zhang, Q.S. ${ }^{a}$

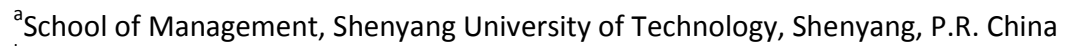 \\ ${ }^{\mathrm{b}}$ School of Management, Bohai University, Jinzhou, P.R. China
}

\begin{abstract}
A B S T R A C T
A production planning model with multiple uncertainties was established in this paper. Customers' demands for quantity, quality, delivery time and price are different. For an ambiguous number of customers, the expectation of the degree of satisfaction was determined by a triangular fuzzy number method. A trapezoidal fuzzy number method was used for customer prices to determine the expectation of satisfaction of the delivery date. Fuzzy intervals and interval numbers were used to describe quality uncertainty and price uncertainty, respectively. A multi-objective planning model was established, which consists of four objectives, namely, meeting customers' needs, minimizing costs, minimizing delivery time and maximizing corporate profits. Then, the non-dominated sorting genetic algorithm (NSGA-II) was implemented to simulate and solve the problem of uncertain optimization. This modal resolved multiple uncertainties in customer demand during the process of production planning for the equipment manufacturing enterprises. The results of the running showed and generated a series of Pareto solutions, which are consistent with the results of a multi-objective planning solution. Manufacturers can obtain the best production plans according to the company's production objective priority rules. Finally, the adaptability and feasibility of the model were verified.
\end{abstract}

\section{ARTICLE INFO}

Keywords:

Production planning;

Multiple uncertainties;

Manufacturing enterprise;

Multi-objective model;

Non-dominated sorting genetic

algorithm (NSGA-II)

*Corresponding author: liuyfmail1979@163.com (Liu, Y.F.)

Article history:

Received 5 June 2018

Revised 26 August 2018

Accepted 7 September 2018

(C) 2018 CPE, University of Maribor. All rights reserved.

\section{References}

[1] Rahdar, M., Wang, L., Hu, G. (2018). A tri-level optimization model for inventory control with uncertain demand and lead time, International Journal of Production Economics, Vol. 195, 96-105, doi: 10.1016/j.ijpe.2017.10.011.

[2] Ho, J.-W., Fang, C.-C. (2013). Production capacity planning for multiple products under uncertain demand conditions, International Journal of Production Economics, Vol. 141, No. 2, 593-604, doi: 10.1016/j.ijpe.2012.09.016.

[3] Shi, J., Zhang, G., Sha, J. (2011). Optimal production planning for a multi-product closed loop system with uncertain demand and return, Computers \& Operations Research, Vol. 38, No. 3, 641-650, doi: 10.1016/i.cor.2010. $\underline{08.008 .}$

[4] Diabat, A., Dehghani, E., Jabbarzadeh, A. (2017). Incorporating location and inventory decisions into a supply chain design problem with uncertain demands and lead times, Journal of Manufacturing Systems, Vol. 43, Part 1, 139-149, doi: 10.1016/i.jmsy.2017.02.010.

[5] Pan, F., Nagi, R. (2010). Robust supply chain design under uncertain demand in agile manufacturing, Computers \& Operations Research, Vol. 37, No. 4, 668-683, doi: 10.1016/i.cor.2009.06.017.

[6] Erdogan, S.A., Denton, B. (2013). Dynamic appointment scheduling of a stochastic server with uncertain demand, INFORMS Journal on Computing, Vol. 25, No. 1, 116-132, doi: 10.1287/ijoc.1110.0482.

[7] Cho, S.-H., Tang, C.-S. (2013). Advance selling in a supply chain under uncertain supply and demand, Manufacturing \& Service Operations Management, Vol. 15, No. 2, 305-319, doi: 10.1287/msom.1120.0423. 
[8] Aghezzaf, E.-H., Sitompul, C., Najid, N.M. (2010). Models for robust tactical planning in multi-stage production systems with uncertain demands, Computers \& Operations Research, Vol. 37, No. 5, 880-889, doi: 10.1016/i.cor. 2009.03.012.

[9] Chica, M., Cordón, Ó., Damas, S., Bautista, J. (2013). A robustness information and visualization model for time and space assembly line balancing under uncertain demand, International Journal of Production Economics, Vol. 145, No. 2, 761-772, doi: 10.1016/j.ijpe.2013.05.030.

[10] Chica, M., Bautista, J., Cordón, Ó., Damas, S. (2016). A multiobjective model and evolutionary algorithms for robust time and space assembly line balancing under uncertain demand, Omega, Vol. 58, 55-68, doi: 10.1016/ j.omega.2015.04.003.

[11] Xu, W., Liu, L., Zhang, Q., Wang, X. (2017). A multi-object decision-making method for location model of manufacturing industry under uncertain environment, Journal of Interdisciplinary Mathematics, Vol. 20, No. 4, 1019-1028, doi: 10.1080/09720502.2017.1358879.

[12] Xu, W., Yu, Y., Zhang, Q. (2018). A multiobjective decision-making method of service element allocation of customer behaviour, Discrete Dynamics in Nature and Society, Vol. 2018, Article ID 3572094, doi: 10.1155/2018/ 3572094.

[13] Tang, M., Gong, D., Liu, S., Lu, X. (2017). Finding key factors affecting the locations of electric vehicle charging stations: A simulation and ANOVA approach, International Journal of Simulation Modelling, Vol. 16, No. 3, 541554, doi: 10.2507/IJSIMM16(3)C015.

[14] Tang, M., Gong, D., Liu, S., Zhang, H. (2016). Applying multi-phase particle swarm optimization to solve bulk cargo port scheduling problem, Advances in Production Engineering \& Management, Vol. 11, No. 4, 299-310, doi: 10.14743/apem2016.4.228.

[15] Galal, N.M., El-Kilany, K.S. (2016). Sustainable agri-food supply chain with uncertain demand and lead time, International Journal of Simulation Modelling, Vol. 15, No. 3, 485-496, doi: 10.2507/IJSIMM15(3)8.350.

[16] Ben Said, L., Hmiden, M., Ghedira, K. (2010). A two-step transshipment model with fuzzy demands and service level constraints, International Journal of Simulation Modelling, Vol. 9, No. 1, 40-52, doi: 10.2507/IJSIMM09(1) 4.142.

[17] Liu, Y.F., Zhang, Q.S. (2018). Solving multi-objective planning model for equipment manufacturing enterprises with dual uncertain demands using NSGA-II algorithm, Advances in Production Engineering \& Management, Vol. 13, No. 2, 193-205, doi: 10.14743/apem2018.2.284.

[18] Fallah-Mehdipour, E., Haddad, O.B., Tabari, M.M.R., Mariño, M.A. (2012). Extraction of decision alternatives in construction management projects: Application and adaptation of NSGA-II and MOPSO, Expert Systems with Applications, Vol. 39, No. 3, 2794-2803, doi: 10.1016/j.eswa.2011. 08.139.

[19] Huang, B., Buckley, B., Kechadi, T.-M. (2010). Multi-objective feature selection by using NSGA-II for customer churn prediction in telecommunications, Expert Systems with Applications, Vol. 37, No. 5, 3638-3646, doi: 10.1016/j.eswa.2009.10.027.

[20] Tang, M., Gong, D., Liu, S., Lu, X. (2017). Finding key factors affecting the locations of electric vehicle charging stations: A simulation and ANOVA approach, International Journal of Simulation Modelling, Vol. 16, No. 3, 541554, doi: 10.2507/IJSIMM16(3)C015. 


\section{APEM}

\title{
Večkriterijski model za načrtovanje proizvodnje za proizvajalce opreme $z$ negotovimi zahtevami
}

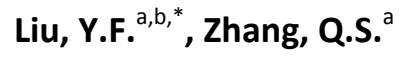 \\ ${ }^{a}$ School of Management, Shenyang University of Technology, Shenyang, P.R. China \\ ${ }^{b}$ School of Management, Bohai University, Jinzhou, P.R. China
}

\section{POVZETEK}

$\mathrm{V}$ članku je opisana vzpostavitev modela načrtovanja proizvodnje z več negotovostmi. Zahteve strank glede količine, kakovosti, dobavnega roka in cene so različne. Za nedoločeno število kupcev je bila pričakovana stopnja zadovoljstva določena z metodo mehkih trikotnih števil. Za določitev pričakovanega zadovoljstva glede dobavnega roka, je bila pri cenah za stranke uporabljena metoda mehkih trapeznih števil. Za opis negotovosti glede kakovosti in cene so bili uporabljeni mehki intervali in intervalna števila. Vzpostavljen je bil večkriterijski model načrtovanja, ki ga sestavljajo štirje kriteriji, in sicer zadovoljevanje potreb strank, zmanjševanje stroškov, skrajšanje dobavnih rokov in povečanje dobička podjetij. Za simulacijo in reševanje problema negotove optimizacije je bil uporabljen genetski algoritem z nedominiranim sortiranjem (NSGA-II). Model je rešil številne negotovosti povezane z zahtevami kupcev, ki se pojavijo med postopkom načrtovanja proizvodnje v podjetjih, ki proizvajajo opremo. Rezultati so prikazani na vrsti Pareto grafov, ki so skladni z rezultati večkriterijskega načrtovanja. Proizvajalci lahko izberejo najboljše proizvodne načrte v skladu s prednostnimi pravili podjetja. Preverjeni sta bili tudi prilagodljivost in izvedljivost modela.

\author{
PODATKI O ČLANKU \\ Ključne besede: \\ Načrtovanje proizvodnje; \\ Številne negotovosti; \\ Proizvodna podjetja; \\ Večkriterijski model; \\ Genetski algoritem z nedominira- \\ nim sortiranjem (NSGA-II) \\ *Kontaktna oseba: \\ liuyfmail1979@163.com \\ (Liu, Y.F.) \\ Zgodovina članka: \\ Prejet 5. junija 2018 \\ Popravljen 26. avgusta 2018 \\ Sprejet 7. septembra 2018
}

